\title{
Irreversible Performance Characteristics of Air Standard Otto Cycles with Polytropic Processes
}

\author{
Mahmoud Huleihili,2* and Gedalya Mazor ${ }^{2}$ \\ ${ }^{1}$ Academic Institute for Training Arab Teachers (AITAT), Beit Berl College, Doar Beit Berl, 44905, Israel \\ ${ }^{2}$ Department of Mechanical Engineering, The Sami Shamoon College of Engineering, 84 Jabotinsky St. Ashdod 77245, Israel
}

\begin{abstract}
In this study, the standard Otto cycle is reconsidered, leading to the replacement of its reversible adiabatic processes by polytropic processes. Two models were proposed, the sub-k polytropic model and the super-k polytropic model, in order to account for the losses encountered in the real Otto engine. The performance characteristics of the Otto engine were presented via plots of power vs. efficiency curves. The results, based on the two models, predict a slightly higher than $30 \%$ reduction in the net power output. The results also show that the efficiency of the real Otto engine lies at approximately $70 \%$ of the efficiency obtained from the standard Otto cycle. The current study's value is generally educational, and may also serve as guidance for engineers performing detailed simulations or conducting expensive experiments in the field.
\end{abstract}

Keywords: Irreversible otto cycle; Polytropic process; Isentropic process

\section{Introduction}

Earth's increasing population, combined with the ongoing depletion of natural resources, dictates serious challenges for scientists, engineers and policy makers. Sustainability is a key concern for today's society, and one way to approach it is through energy efficiency, including increasing the efficiency of power systems, which may help reduce pollution.

Internal combustion engines [1], used in transportation systems worldwide, are a major source of carbon dioxide emissions. For example, according to Taylor in the UK such engines produce about $23 \%$ of the country's total carbon dioxide emission, up from $14 \%$ in 1980 [2]. Current scientific advancements in the field suggest the possibility of a $6-15 \%$ improvement in internal combustion fuel efficiency in the coming decade, although the filters required by emission legislation in various countries reduce these gains. In hybrid cars based on both internal combustion engines and electric motors, energy requirements are reduced in the order of $21-28 \%$. The developments beyond the next decade are likely to be dominated by four topics: emission legislation and emission control, new fuels, improved combustion, and a range of advanced concepts for saving energy. Emission control is important because current methods for limiting nitrogen oxides and particulate emissions imply extra energy consumption [2].

The internal combustion engine, Taylor is modeled with the Otto cycle as a limiting upper bound [1]. The Otto cycle includes four branches: two isochors and two reversible adiabatic processes. The adiabatic processes may be replaced by two polytropic processes to account for the deviations from the ideal branches. This serves as a way to account for losses that occur in the internal combustion engine, such as heat leaks and losses, friction, and incomplete combustion [1].

Various studies have investigated the feasibility and potential advantages of small scale Miller cycle natural gas engines for applications such as domestic heat power systems. In their model of such a Miller cycle engine, Mikalsen et al. [3] assumed that the in-cylinder gases follow polytropic compression and expansion state changes. Among the various thermodynamic parameters that affect the Otto engine work output is the engine's pressure [3]. In a different study, AnguloBrown et al. (1994) [4] proposed an irreversible simplified model for the air standard Otto thermal cycle. Their model took into account the finite-time evolution of the cycle's compression and power strokes and lumped global losses into a friction-like term. Angulo-Brown's model also permitted the maximization of quantities such as power output and efficiency in terms of the compression ratio $r$. The optimum $r$ values obtained from the model compared favorably to the standard $r$ values for real Otto engines. Furthermore, the Angulo-Brown model leads to loop-shaped power vs. efficiency curves, as is common to almost all real heat engines, and as demonstrated previously for the thermoelectric heat engine [5] and for different classes of heat engines [6]. Aiming to account for losses accrued in internal combustion engines, Zhao and Chen [7] established an irreversible cycle model of the Miller heat engine, in which the multi-irreversibilities coming from the adiabatic compression and expansion processes, finite time processes and heat leak loss through the cylinder wall are taken into account. In addition, the power output and efficiency of the cycle were optimized with respect to the pressure ratio of the working substance. Zhao and Chen [7] also discussed the optimum criteria of certain important parameters, such as the power output, efficiency and pressure ratio, as well as the influence of some relevant design parameters. Moreover, the authors suggested that the Otto and Atkinson heat engines may be taken as two special cases of the Miller heat engine, and that the optimal performance of both heat engines may be directly derived from that of the Miller heat engine [7].

The methods of finite-time thermodynamics as was suggested by Curzon and Ahlborn [8] have been previously used to examine the performance of an air standard Atkinson cycle (a type of internal combustion engine) with heat-transfer loss, friction-like term loss and variable specific-heats of the working fluid. Ge et al. [9] derived

*Corresponding author: Mahmoud Huleihil, Academic Institute for Training Arab Teachers (AITAT), Beit Berl College, Doar Beit Berl, 44905, Israel, Tel: 097476333; Fax: 09-7454104; E-mail: cs.berl@gmail.com

Received June 27, 2012; Accepted August 30, 2012; Published September 03, 2012

Citation: Huleihil M, Mazor G (2012) Irreversible Performance Characteristics of Air Standard Otto Cycles with Polytropic Processes. J Appl Mech Eng 1:111. doi:10.4172/2168-9873.1000111

Copyright: @ 2012 Huleihil M, et al. This is an open-access article distributed under the terms of the Creative Commons Attribution License, which permits unrestricted use, distribution, and reproduction in any medium, provided the original author and source are credited. 
several relations via numerical examples: the relation between power output and the compression ratio; between thermal efficiency and the compression ratio; and between power output and the efficiency of the cycle. Moreover, the authors analyzed the effects of variable specific-heats of the working fluid and the friction-like term loss on the irreversible cycle performance [9]. Their results showed that the analysis of the cycle should consider the effects of variable specific-heats of working fluid and friction-like term loss on the irreversible cycle's performance. The results obtained in the study also provide guidance for the design of Atkinson engines [9]. Hernandez et al. [10] addressed the performance characteristic of the internal combustion engine via a simplified model for an irreversible Otto cycle, which accounted for the characteristic power vs. efficiency curve of real heat engines [10]. Their model avoided the usual hypotheses of endoreversible heat engines, as it considered an air Otto engine with internally dissipative friction and used pure sinusoidal law to describe the piston velocity on the adiabatic branches [10]. Another study by Angulo-Brown et al. [11] proposed a finite-time thermodynamics model for an Otto thermal cycle [11]. Their model considered global losses in a simplified manner, by lumping them into a friction-like term, and took into account the departure from an endoreversible regime through a parameter $(\mathrm{R})$ arising from the Clausius inequality. The model's numerical results suggest that the cycle's power output and efficiency are very sensitive to the $\mathrm{R}$ parameter. Moreover, the authors found that $\mathrm{R}$ is the ratio of the constant-volume heat capacities of the reactants and products in the combustion reaction occurring inside the working fluid. Such results have implications for the search for new fuels for internal combustion engines [11]. In her 1996 study, Kuo attempted to predict the gas pressure changes within the cycle of a spark ignition engine using thermodynamic principles [12]. Kuo's model took into account several processes, including compression, with a polytropic exponent of 1.3, and expansion, with a polytropic exponent of 1.25 [12]. Further, Heywood reported that for both the compression and expansion processes, the polytropic exponent $n$ is 1.3 for conventional fuels [13]. In his recent study, Reza [14] stated that in typical internal combustion engines, thousands of cycles are performed per minute. In this sequence of cycles, many physical and chemical quantities change from cycle to cycle for example, the combustion heat changes due to residual gases, imperfect combustion and other reasons [14], such as pressure drops along the constant volume branches [15]. Furthermore, the Otto cycle which serves as an important model of the Otto engine was considered further and extensively under different study assumptions [16-49].

In the current study, the Otto engine is reconsidered, with a focus on the polytropic power $n$. The reversible adiabatic processes in the standard Otto cycle are replaced by irreversible polytropic processes accounting for the aforementioned losses in the spark ignition internal combustion engine (real Otto cycle). Two models, the sub-k polytropic model and the super-k polytropic model, are considered to account for these losses.

The article is constructed as follows: Section 1 consists of the introduction, and Section 2 considers the standard Otto cycle with polytropic branches. The results, including numerical examples, are presented in section 3. Finally, Section 4 give details about the study's conclusions and related discussion.

\section{The Standard Otto Cycle with Polytropic Branches}

\section{The standard Otto cycle}

In this subsection, the standard Otto cycle is briefly reviewed for the convenience of the reader and for ease of reference. For an ideal gas with the given initial conditions (temperature, pressure and volume) and with known maximum temperature and minimum volume, it is possible to define all other states based on the ideal gas laws and the various processes which make up the thermodynamic cycle. Based on the first law of thermodynamics, it is possible to calculate the heat input, heat rejected and network output obtained in given cycle per one kilogram of gas. The efficiency is defined as the ratio of the network output divided by the heat input to the engine. Specifically, for a known initial temperature $T_{1}(\mathrm{~K})$, initial pressure $p_{1}(\mathrm{~Pa})$, initial volume (maximum) $V_{1}\left(\mathrm{~m}^{3}\right)$, minimum volume $V_{2}\left(\mathrm{~m}^{3}\right)$ and the maximum temperature in the cycle $T_{3}(\mathrm{~K})$, one can obtain the temperature of an adiabatic process at the end of the compression process $T_{2}(\mathrm{~K})$. This temperature is given as a function of the compression ratio $\left(r=V_{1} / V_{2}\right)$ by:

$$
T_{2}=T_{1} r^{k-1}
$$

Similarly, the temperature at the end of the expansion process $\mathrm{T}_{4}$ is given by:

$$
T_{4}=T_{3} / r^{k-1}
$$

The first law of thermodynamics, for any process which defines a relation between heat $Q(\mathrm{~J})$, work $W(\mathrm{~J})$ and change in internal energy $\Delta E(\mathrm{~J})$ is given by:

\section{$Q=\Delta E+W$}

Equations (1)-(3), along with the known minimum and maximum temperatures are used to define the heat input to the engine, per kilogram of the working fluid $Q_{23}$, which is given by:

$$
Q_{23}=C_{v}\left(T_{3}-T_{2}\right)
$$

Where $C_{v}$ is the specific heat at constant volume $(\mathrm{J} / \mathrm{kg} / \mathrm{K})$. Similarly, the heat rejection from engine $Q_{41}$ is given by:

$$
Q_{41}=C_{v}\left(T_{4}-T_{1}\right)
$$

Heat quantities must be defined as positive entities. The work output is given by:

$$
W=Q_{23}-Q_{41}
$$

Finally, the thermal efficiency $\eta$ of the engine is defined by:

$$
\eta=\frac{W}{Q_{23}}
$$

\section{The sub-k polytropic model}

The Standard Otto cycle is depicted in figure 1, following the different branches along the cycle 1-2-3-4-1. 1-2 refers to the reversible

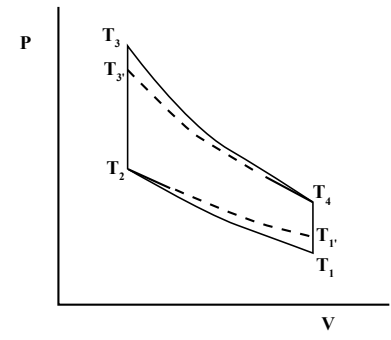

Figure 1: Schematic drawings of Model 1 (sub-k model), describing the irreversible standard Otto cycle with polytropic branches instead of reversible adiabatic branches. Values of the polytropic exponent are smaller than the ratio of heat capacities at constant pressure and constant volume. 


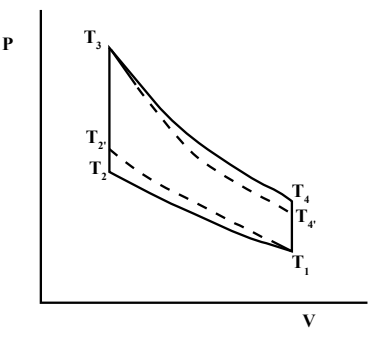

Figure 2: Schematic drawings of Model 2 (super-k model), describing the irreversible standard Otto cycle with polytropic branches instead of reversible adiabatic branches. Values of the polytropic exponent are greater than the ratio of heat capacities at constant pressure and constant volume.

adiabatic process, 2-3 to constant volume heat addition, 3-4 to reversible adiabatic expansion, and 4-1 to constant volume heat rejection. The modified cycle, in the sub-k polytropic model is traced as follows: 1'-23'-4-1'. The divergences from the ideal cycle are expressed as a) cooling down to point 1',which is higher than point 1 , and then heating to point 2 in order to maintain temperature $T_{2}$. This dictates heat rejection along polytropic process 1 '-2, and $b$ ) heat is added to increase the temperature up to point 3', which is lower than point 3; afterwards, heat must be added along the expansion process 3'-4 in order to maintain the temperature $T_{4}$. As shown in figure 1 , the net work output is degraded as compared to the standard cycle. It will be shown that the efficiency is reduced as well. For the polytropic process 1'-2, the temperature $T_{2}$ is given by:

$$
T_{2}=T_{1} r^{n-1}
$$

Based on Equations (1) and (8), it is possible to express $T_{1}$, as follows:

$$
T_{1}=T_{1} r^{k-n}
$$

Equation (2) can be reversed after appropriate changes are made to calculate $T_{3}$, given by:

$$
T_{3}=T_{4} r^{n-1}
$$

After substitution of $T_{4}$ Equation (2) into Equation (10), the temperature $T_{3}$, is given by:

$$
T_{3}=T_{3} r^{n-k}
$$

Taken together, Equations (9), (11) and figure 1 show that the value of $n$ must be smaller than $k$.

For a polytropic process with $p V^{n}=$ const along a process a-b, the amount of heat transfer is given by:

$$
Q_{a b}=c_{v} \frac{k-n}{n-1}\left(T_{b}-T_{a}\right)
$$

After the temperatures and types of processes are known, the amount of heat input or output may be calculated. Along branch 1'-2, polytropic compression, the amount of heat rejected $Q_{1^{\prime} 2}$ is given by:

$$
Q_{1^{\prime} 2}=c_{v} \frac{k-n}{n-1}\left(T_{2}-T_{1^{\prime}}\right) \text {, }
$$

The heat added in the constant volume process $Q_{23}$, is given by:

$Q_{23}=C_{v}\left(T_{3^{\prime}}-T_{2}\right)$,

The heat added at the polytropic expansion process $Q_{3^{\prime} 4}$ is given by:

$$
Q_{3^{\prime} 4}=c_{v} \frac{n-k}{n-1}\left(T_{4}-T_{3^{\prime}}\right)
$$
by:

Finally, the heat rejected at the constant volume process $Q_{41}$, is given

$$
Q_{41^{\prime}}=c_{v}\left(T_{4}-T_{1^{\prime}}\right)
$$

Based on Equation (6) along with Equations (13)-(16), the net work output $W_{1}$ for the first model may be calculated and given by:

$$
W_{1}=\left(Q_{23^{3}}+Q_{3^{\prime} 4}\right)-\left(Q_{1^{\prime 2}}+Q_{41}\right)
$$

Lastly, the thermal efficiency of the first model $\eta_{1}$ is given by:

$$
\eta_{1}=\frac{W_{1}}{Q_{23}+Q_{34}}
$$

\section{The super-k polytropic model}

In the second model, the super-k polytropic model (Figure 2), the losses in the internal combustion engine are lumped into polytropic process 3-4' (polytropic expansion to a lower point 4', below point 4) and polytropic process 1-2' (polytropic compression to a higher point 2 ' which is above point 2). Both processes indicate that heat is lost along path 3-4' and added along path 1-2'. Again, in this cycle both net work output and efficiency are degraded compared to their values in the ideal cycle. When the minimum and maximum temperatures and the polytropic processes are known, the temperatures at the end of each process may be calculated. Specifically, the temperature at the end of the polytropic process is given by:

$$
T_{2^{\prime}}=T_{1} r^{n-1}
$$
by:

And the temperature at the end of the polytropic expansion is given

$$
T_{4^{\prime}}=T_{3} / r^{n-1}
$$

By observing equations (19) and (20), it may be deduced that the values of $n$ must be greater than $k$. Next, based on equation (12) along with the first law of thermodynamics and equations (19) and (20), the heat transfer amounts are calculated. The heat input along the polytropic compression process $Q_{12}$, is given by:

$Q_{12^{\prime}}=c_{v} \frac{n-k}{n-1}\left(T_{2^{\prime}}-T_{1}\right)$,

The heat input along the constant volume process $Q_{2^{\prime} 3}$ is given by:

$Q_{2^{\prime} 3}=C_{v}\left(T_{3}-T_{2^{\prime}}\right)$

Heat rejection along the polytropic expansion process $Q_{34}$, is given by:

$Q_{34^{\prime}}=c_{v} \frac{n-k}{n-1}\left(T_{3}-T_{4^{\prime}}\right)$

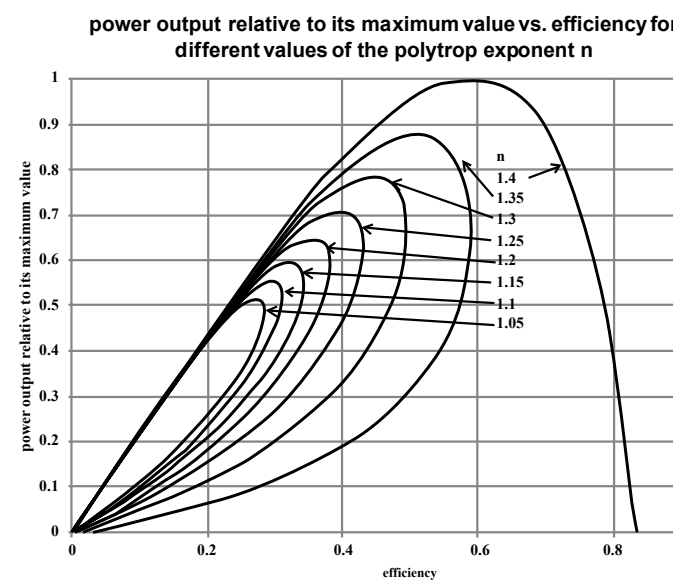

Figure 3: Power output (work output per cycle time) relative to its maximum value vs. efficiency for different values of the polytropic exponent $n$, based on model 1. 
Citation: Huleihil M, Mazor G (2012) Irreversible Performance Characteristics of Air Standard Otto Cycles with Polytropic Processes. J Appl Mech Eng 1:111. doi:10.4172/2168-9873.1000111

Page 4 of 6

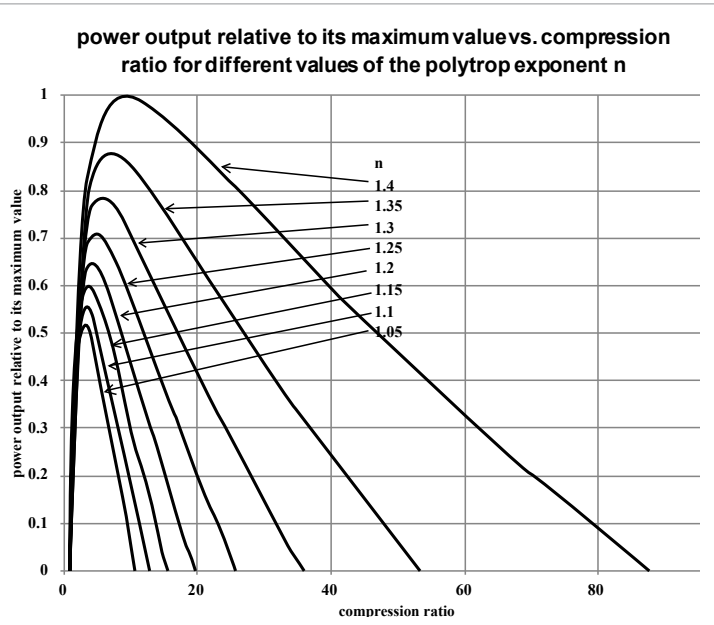

Figure 4: Power output (work output per cycle time) relative to its maximum value vs. compression ratio $r$ for different values of the polytropic exponent $n$, based on model 1.

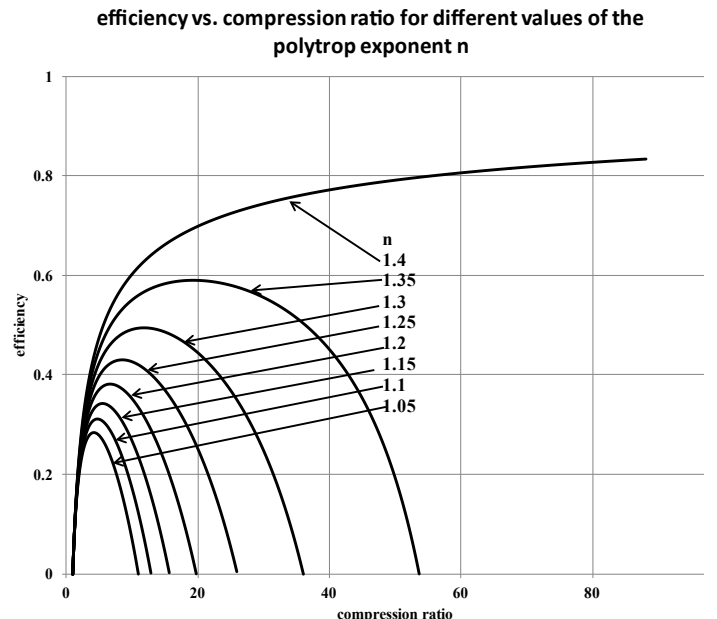

Figure 5: Efficiency vs. compression ratio $r$ for different values of the polytropic exponent $n$ based on model 1 .

Finally, heat rejection along the constant volume branch $Q_{4^{\prime} 1}$ is given by:

$$
Q_{2^{\prime} 3}=C_{v}\left(T_{3}-T_{2^{\prime}}\right)
$$

When all heat transfer quantities are known, the net work may be equated with output $W_{2}$ for the second model, which is given by:

$$
W_{2}=\left(Q_{12^{\prime}}+Q_{22^{\prime} 3}\right)-\left(Q_{34^{\prime}}+Q_{4^{\prime} 1}\right)
$$

The efficiency for the second model is given by:

$$
\eta_{2}=\frac{W_{2}}{Q_{12^{\prime}}+Q_{2^{\prime} 3}}
$$

Crucially, when $\mathrm{n}=\mathrm{k}$ for both models considered above, the net work output and efficiency expressions coincide with appropriate expressions of the standard cycle. Specifically, the net work output $W$ for the standard cycle is given by:

$$
W=c_{v} T_{3}\left(1-\frac{T_{1}}{T_{3}} r^{k-1}\right)\left(1-\frac{1}{r^{k-1}}\right)
$$

Finally, the efficiency $\eta$ for the standard Otto cycle is given by the following:

$$
\eta=1-\frac{1}{r^{k-1}}
$$

\section{Results and Numerical Examples}

The maximum temperature $T_{3}$ was assumed to be $1800 \mathrm{~K}$, based on the equilibrium product temperatures for the constant-volume process (1800 K represents the mean value between $800 \mathrm{~K}$ and $2800 \mathrm{~K}$ ) [13]. The minimum temperature $T$, was actually the ambient temperature, approximately $300 \mathrm{~K}$. Accordingly, the ratio $T_{1} / T_{3}=1 / 6$. These values were needed to create plots of efficiency $v s$. compression ratio, power (work output per cycle time) relative to its maximum value, and power $v s$. efficiency for different values of the polytropic exponent $n$. The aforementioned plots (Figures 3-8) were prepared for both models described in the previous section. The values of the polytropic exponents in general attain different values for the expansion process $\left(n_{e}\right)$ and for the compression processes $\left(n_{c}\right)$. The effect of these different values is presented in figure 9 .

In order to compare the predicted results of the two models, a sensitivity study was performed. For a typical value -8 of the compression ratio of the Otto engine [13], the efficiency and work



Figure 6: Power output (work output per cycle time) relative to its maximum value vs. efficiency for different values of the polytropic exponent $n$ based on model 2.

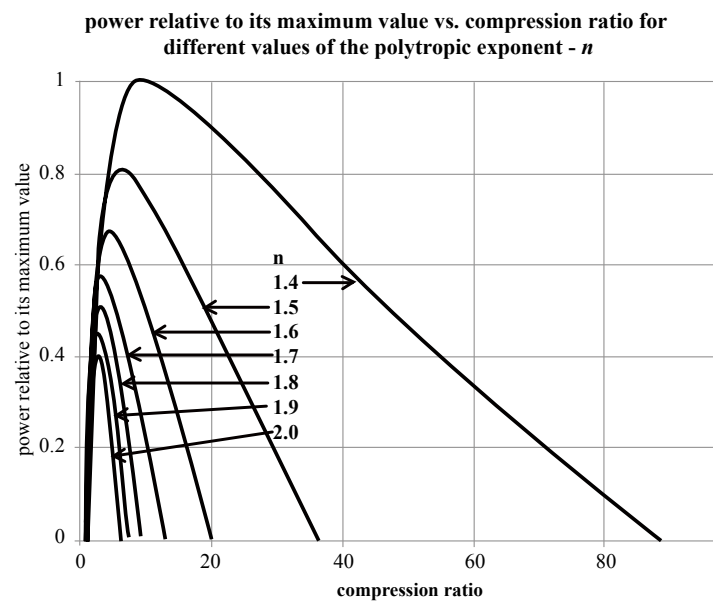

Figure 7: Power output (work output per cycle time) relative to its maximum value vs. compression ratio $r$ for different values of the polytropic exponent $n$ based on model 2 . 


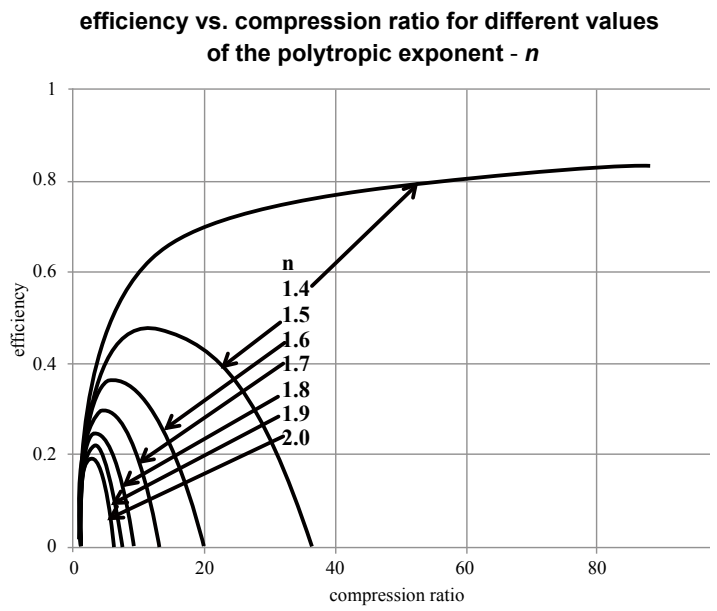

Figure 8: Efficiency vs. compression ratio $r$ for different values of the polytropic exponent $n$ based on model 2.

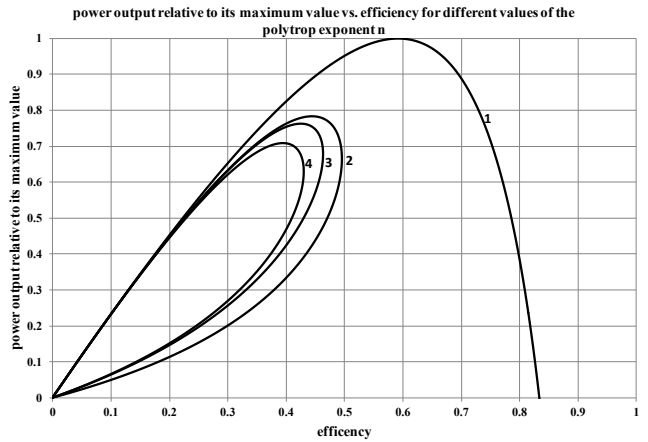

Figure 9: Power output (work output per cycle time) relative to its maximum value vs. efficiency for different values of the polytropic exponent $n$, based on model 1. The numbers to the right of the lines in the plot indicate the following: 1 ) the polytropic exponent for expansion $(n)$ and for compression $\left(n_{c}\right)$ have the same value and both are equal to $\left.k ; 2\right) n_{e}=n_{c}=1.3 ; 3-n_{e}=1.3$ $n_{c}=1.25 ; 4-n_{e}=n_{c}=1.25$.

output were compared to the results predicted by the ideal Otto cycle. The calculations were performed for $n=k \pm 10 \% k(1.25-1.4-1.55)$. The predicted results for the sub-k polytropic model showed a reduction of $35 \%$ in work output and $24 \%$ in efficiency. The super-k polytropic model predicted similar but slightly different results: a 35\% reduction in work output and a $72 \%$ reduction in efficiency. Taken together, these results show that the efficiency of real engines is slightly higher than the $70 \%$ efficiency rate predicted by the ideal cycle. A similar observation has been well-documented for the comparison of real engine efficiency and Carnot efficiency.

\section{Summary and Conclusions}

In this article, the standard Otto cycle is briefly reviewed, in order to serve as a basis for two proposed irreversible Otto cycles.

In the first model (the sub-k polytropic model), the reversible adiabatic branches were replaced with polytropic branches with power $n$ less than $k$. The reversible adiabatic compression branch was replaced by a polytropic compression branch. As the starting temperature of the sub-k polytropic model is higher than the minimum temperature of the standard Otto cycle, heat must be rejected from the irreversible cycle in order to reach the same standard temperature by the end of the compression process. As a result of the heat addition to the constant volume process, and accounting for losses along this branch, the temperature at the end of the process reaches a maximum which is slightly lower than the maximum temperature in the standard Otto cycle, as depicted in Figure 1. Similarly, the reversible adiabatic branch is replaced by a polytropic branch, and heat input is required to attain the same temperature by the end of the adiabatic process of the standard cycle. Finally, heat is rejected by the constant volume branch, thus completing the polytropic cycle.

In the second model (the super- $\mathrm{k}$ polytropic model), the irreversibilities are accounted for by replacing the adiabatic branches as was done in the sub-k polytropic model, but with values of $n$ greater than $k$. Thus, heat must be added at the compression process while being rejected at the expansion process.

Heat balance equations, based on the first law of thermodynamics, were used to calculate the network output and thermodynamic efficiency of each model. The results were displayed by a series of plots (Plots 3-9) for a typical case, with a minimum temperature of $300 \mathrm{~K}$ and a maximum temperature of $1800 \mathrm{~K}$. The two models were then compared for a variation of $10 \%$ around the air value $k=1.4$. Both models predicted similar results: a reduction of a slightly more that $30 \%$ in power output and a reduction of slightly less than $30 \%$ in efficiency. In summary, both models predicted that real heat engines' efficiency is slightly larger than the $70 \%$ rate predicted by the ideal cycle.

Finally, the value of such a study might fall in the following categories: 1) Educational, and 2) it could serve as a guidance tool for the engineer before performing detailed simulations or conducting expensive experiments.

\section{References}

1. Taylor CF (1971) The internal-combustion engine in theory and practice. M.I.T Press, Cambridge Massachusetts, USA

2. Taylor AMKP (2008) Science review of internal combustion engines. Energy Policy 36: 4657-4667.

3. Mikalsen R, Wang YD, Roskilly AP (2009) A comparison of Miller and Otto cycle natural gas engines for small scale CHP applications. Applied Energy 86: 922 927.

4. Angulo-Brown F, Fernández-Betanzos J, Diaz-Pico CA (1994) Compression ratio of an optimized air standard Otto-cycle model. Eur J Phys 15: 38

5. Gordon JM, Huleihil M (1992) General performance characteristics of real heat engines. J Appl Phys 72: 829-837.

6. Gordon JM (1991) Generalized power versus efficiency characteristics of heat engines: The thermoelectric generator as an instructive illustration. Am J Phys 59: 551- 555 .

7. Zhao Y, Chen J (2007) Performance analysis of an irreversible Miller heat engine and its optimum criteria. Applied Thermal Engineering 27: 2051-2058.

8. Curzon FL, Ahlborn B (1975) Efficiency of a Carnot Engine at Maximum Power Output. Am J Phys 43: 22-24

9. Ge Y, Chen L, Sun F, Wu C (2006) Performance of an Atkinson cycle with heat transfer, friction and variable specific-heats of working fluid. Applied Energy 83 1210-1221.

10. Hernandez AC, Medina A, Roco JMM, Velasco S (1995) On an irreversible air standard Otto-cycle model. Eur J Phys 16: 73-75.

11. Angulo-Brown F, Rocha-Martínez JA, Navarrete-González TD (1996) A nonendoreversible Otto cycle model: improving power output and efficiency. J Phys D Appl Phys 29: 80-83.

12. Kuo PS (1996) Cylinder pressure in spark-ignition engine: A computationa model. J Undergrad Sci 3: 141-145.

13. Heywood J (1988) Internal Combustion Engine: Fundamentals (ISE). McGraw Hill, New York, USA: 385 
Citation: Huleihil M, Mazor G (2012) Irreversible Performance Characteristics of Air Standard Otto Cycles with Polytropic Processes. J Appl Mech Eng 1:111. doi:10.4172/2168-9873.1000111

14. Nejad RM (2012) Power output and Efficiency of internal combustion engine based on the FTT theory. Life Science Journal 9: 387-390.

15. Huleihil M (2011) Effects of Pressure Drops on the Performance Characteristics of Air Standard Otto Cycle. Physics Research International 1: 1-7.

16. Leff HS (1987) Thermal efficiency at maximum work output: new results for old heat engines. Am J Phys 55: 602-610.

17. Klein SA (1991) An explanation for observed compression ratios in internal combustion engines. Trans ASME J Eng Gas Turbine Pow 113: 511-513.

18. Wu C, Blank DA (1992) The effect combustion on a work-optimized endoreversible Otto cycle. J Energy Inst 65: 86-89.

19. Wu C, Blank DA (1993) Optimization of the endoreversible Otto cycle with respect to both power and mean effective pressure. Energy Conversion and Management 34: 1255-1259.

20. Chen L, Wu C, Sun F, Cao S (1998) Heat transfer effects on the net work output and efficiency characteristics for an air standard Otto cycle. Energy Conversion and Management 39: 643-648.

21. Ficher A, Hoffman KH (2004) Can a quantitative simulation of an Otto engine be accurately rendered by a simple Novikov model with heat leak? J Non-Equilib Thermody 29: 9-28.

22. Ozsoysal OA (2006) Heat loss as a percentage of fuel's energy in air standard Otto and Diesel cycles. Energy Conversion Management 47: 1051-1062.

23. Hou SS (2007) Comparison of performances of air standard Atkinson and Otto cycles with heat transfer considerations. Energy Conversion and Management 48: 1683-1690.

24. Chen L, Zheng T, Sun F, Wu C (2003) The power and efficiency characteristics for an irreversible Otto cycle. International Journal of Ambient Energy 24: 195200

25. Chen J, Zhao Y, He J (2006) Optimization criteria for the important parameters of an irreversible Otto heat-engine. Applied Energy 83: 228-238.

26. Zhao YR, Chen JC (2008) Irreversible Otto heat engine with friction and heat leak losses and its parametric optimum criteria. Journal of the Energy Institute 81: $54-58$.

27. Feidt M (2009) Optimal thermodynamics-New Upperbounds. Entropy 11: 529547.

28. Ebrahimi R (2010) Effects of gasoline-air equivalence ratio on performance of an Otto engine. J Am Sci 6: 131-135.

29. Ebrahimi R (2010) Theoretical study of combustion efficiency in an Otto engine. J Am Sci 6: 113-116.

30. Ozsoysal OA (2010) Effects of combustion efficiency on an Otto cycle. International Journal of Exergy 7: 232-242.

31. Ebrahimi R, Ghanbarian D, Tadayon MR (2010) Performance of an Otto engine with volumetric efficiency. J Am Sci 6: 27-31.

32. Gumus M, Atmaca M, Yilmaz T (2009) Efficiency of an Otto engine under alternative power optimizations. International Journal of Energy and Research 33: $745-752$

33. Ust $Y$ (2005) Ecological performance analysis of irreversible Otto cycle. J Eng Natural Sci 3: 106-117.

34. Mehta HB, Bharti OS (2009) Performance analysis of an irreversible Otto cycle using finite time thermodynamics. Proceedings of the World Congress on Engineering, London, UK.

35. Wu F, Chen L, Sun F, Wu C, Guo F, et al. (2006) Quantum degeneracy effect on performance of irreversible Otto cycle with deal Bose gas. Energy Conversion and Management 47: 3008-3018.

36. Wang H, Liu S, He J (2009) Performance analysis and parametric optimum criteria of a quantum Otto heat engine with heat transfer effects. Applied Thermal Engineering 29: 706-711.

37. Wang H, Liu S, Du J (2009) Performance analysis and parametric optimum criteria of a regeneration Bose-Otto engine. Phys Scr 79: 055004

38. Nie W, Liao Q, Zhang C, He J (2010) Micro-/nanoscaled irreversible Otto engine cycle with friction loss and boundary effects and its performance characteristics. Energy 35: 4658-4662.
39. Wu F, Chen L, Sun F, Wu C, Guo F, et al. (2006) Ecological optimization performance of an irreversible quantum Otto cycle working with an ideal Ferm gas. Open System \& Information Dynamics 13: 55-66.

40. Rocha-Martinez JA, Navarrete-Gonzalez TD, Pava-Miller CG, PaezHernandez R, Angulo-Brown F (2002) Otto and Diesel engine models with cyclic variability. Revista mexicana de física 48: 228-234

41. Rocha-Martinez JA, Navarrete-Gonzalez TD, Pavia-Miller CG, Ramirez-Rojas A, Angulo-Brown F (2006) A simplified irreversible Otto engine model with fluctuations in the combustion heat. International Journal of Ambient Energy 27: 181-192.

42. Ge Y, Chen L, Sun F, Wu C (2005) Thermodynamic simulation of performance of an Otto cycle with heat transfer and variable specific heats of working fluid. Int Journal of Thermal Science 44: 506-511.

43. Ge Y, Chen L, Sun F, Wu C (2005) The effects of variable specific heats of working fluid on the performance of an irreversible Otto cycle. Internationa Journal of Exergy 2: 274-283.

44. Zhao Y, Lin B, Chen J (2007) Optimum criteria on the important parameters of an irreversible Otto heat engine with the temperature-dependent hea capacities of the working fluid. ASME Trans J Energy Res Tech 129: 348-354.

45. Lin JC, Hou SS (2008) Effects of heat loss as percentage of fuel's energy friction and variable specific heats of working fluid on performance of air standard Otto cycle. Energy Conversion and Management 49: 1218-1227.

46. Nejad RM, Marghmaleki IS, Hoseini R, Alaei P (2011) Effects of irreversible different parameters on performance of air standard Otto cycle. J American Sci 7: 248-254

47. Ge Y, Chen L, Sun F (2008) Finite time thermodynamic modeling and analysis for an irreversible Otto cycle. Applied Energy 85: 618-624.

48. Ebrahimi R (2010) Effects of variable specific heat ratio on performance of an endoreversible Otto cycle. Acta Physica Polonica A 117: 887-891.

49. Ebrahimi R (2009) Engine speed effects on the characteristic performance of Otto engines. J Am Sci 6: 123-128. 